\title{
Prevalência de hipotireoidismo em pacientes com diabetes mellitus tipo 2
}

\author{
Prevalence of hypothyroidism in patients with type 2 diabetes
}

Gabriel Queiroz Fernandes', Giordanne Guimarães Freitas²

Fernandes GQ, Freitas GG. Prevalência de hipotireoidismo em pacientes com diabetes mellitus tipo 2 / Prevalence of hypothyroidism in patients with type 2 diabetes. Rev Med (São Paulo). 2018 maio-jun.;97(3):273-7.

RESUMO: Introdução. A diabetes mellitus tipo 2 é uma síndrome clínica que torna o corpo incapaz de normalizar os níveis séricos de glicose. O hipotireoidismo, por sua vez, reflete uma condição em que há um baixo nível de hormônios tireoidianos no corpo. A relação entre diabetes mellitus tipo 1 e o hipotireoidismo já é bem estabelecida, mas sua relação com o tipo 2 ainda não é bem descrita na comunidade acadêmica. Casuística e Métodos. Foram incluídos nesta pesquisa pacientes atendidos entre janeiro de 2015 e dezembro de 2016, que possuíam diagnóstico de diabetes mellitus tipo 2. Os instrumentos de coleta foram: ficha clínica com dados sobre perfil e identificação do paciente, inventário de saúde, aspectos clínicos do motivo da consulta, avaliação física, avaliação laboratorial, diagnóstico médico e plano de tratamento. Foram selecionados 184 prontuários médicos de pacientes atendidos no período em questão, sendo que foram excluídos 31 pacientes devido à falta de dados necessários para categorização. Foram revisados os prontuários médicos dos 153 pacientes restantes, sendo que apenas 51 possuíam diagnóstico de diabetes mellitus tipo 2 e foram incluídos nesta pesquisa. Resultados. Após a revisão dos prontuários médicos, 15 pacientes foram diagnosticados com hipotireoidismo, determinando uma prevalência de 29,4\%. Discussão. A alta prevalência encontrada sugere que o rastreio para hipotireoidismo em diabéticos tipo 2 pode levar ao diagnóstico precoce. Conclusão. A alta prevalência de hipotireoidismo estabelecida neste estudo leva a recomendação de rastreio para essa doença em todos os pacientes diabéticos tipo 2.

Descritores: Diabetes mellitus tipo 2; Poliendocrinopatias autoimunes; Hipotireoidismo/epidemiologia; Prevalência; Epidemiologia.
ABSTRACT: Introduction. Type 2 diabetes mellitus is a clinical syndrome that makes the body unable to normalize serum glucose levels. Hypothyroidism reflects a condition in which there is a low level of thyroid hormones in the body. The relation between type 1 diabetes mellitus and hypothyroidism is already well established, but its relation to type 2 is still not well described in the academic community. Casuistry and Methods. Were included in this study patients treated between January 2015 and December 2016, who had a diagnosis of type 2 diabetes mellitus. The instruments of collection were: clinical form with data about profile and patient identification, health inventory, clinical aspects of the reason of the medical appointment, physical evaluation, laboratory evaluation, medical diagnosis and treatment plan. Were selected 184 medical records of patients attended in this period, been that 31 patients were excluded due to the lack of data needed for categorization. The medical records of the 153 remaining patients were reviewed, of which only 51 had a diagnosis of type 2 diabetes mellitus and were included in this study. Results. After reviewing the remaining medical records, 15 patients were diagnosed with hypothyroidism, determining a prevalence of $29.4 \%$. Discussion. The high prevalence found suggests that screening for hypothyroidism in type 2 diabetics may lead to early diagnosis. Conclusion. The high prevalence of hypothyroidism established in this study leads to the recommendation of screening for this disease in all type 2 diabetic patients.

Keywords: Diabetes mellitus, type 2; Hypothyroidism/ epidemiology; Polyendocrinopathies, autoimmune; Prevalence; Epidemiology.

1. Universidade de Rio Verde - UniRV, Faculdade de Medicina, Rio Verde, GO, BR. Graduando em Medicina. ORCID: http://orcid. org/0000-0002-5205-7688. Email: gabrielqfs@gmail.com.

2. Universidade de Rio Verde - UniRV, Faculdade de Medicina, Rio Verde, GO, BR. Centro de Especialidades Médicas. Orientador. Médico endocrinologista. ORCID: http://orcid.org/0000-0001-5970-0800. Email: giordanne@uol.com.br.

Endereço para correspondência: Gabriel Q. Fernandes. Rua Boulevard Amendoeiras, QD 76, LT 01, CS 02, Jardim Atlântico, Goiânia, Goiás, Brasil. CEP 74.343-030 


\section{INTRODUÇÃO}

Organização Mundial de Saúde (OMS) define diabetes como uma doença crônica que ocorre devido a uma produção insuficiente de insulina pelo pâncreas ou a uma incapacidade do corpo em utilizar de modo eficaz a insulina produzida. Em 2017, 422 milhões de adultos no mundo tinham diabetes, sendo essa doença responsável diretamente por 1,6 milhão de mortes anuais ${ }^{1}$.

Dentre os principais fatores de risco para diabetes estão a história familiar de diabetes, o Índice de Massa Corporal (IMC) elevado (obesidade), tabagismo, valores elevados de enzimas hepáticas, níveis reduzidos na secreção ou redução da ação periférica da insulina (resistência a insulina), sedentarismo, alimentação rica em gorduras e baixa ingestão de fibras alimentares ${ }^{2-3}$.

Há vários tipos de diabetes, com etiologias variadas. Os dois tipos mais comuns são: diabetes mellitus tipo $1 \mathrm{e}$ tipo 2. A diabetes mellitus tipo 2 (DMt2) é caracterizada por uma produção insuficiente de insulina, e/ou uma alteração na ação periférica da insulina, tornando o corpo incapaz de normalizar os níveis séricos de glicose ${ }^{2}$. A diabetes mellitus tipo 1 (DMt1) é uma doença autoimune, em que a insulina não é produzida devido a destruição das células $\beta$ pancreáticas, que são responsáveis pela produção pancreática de insulina. A insulina, produzida pelas células $\beta$ pancreáticas, é um dos principais hormônios anabólicos do corpo humano, sendo que sua principal função é facilitar a captação da glicose pelas células musculares estriadas e pelos adipócitos, que juntos representam dois terços da massa corporal ${ }^{4}$.

O hipotireoidismo, por sua vez, atua como um fator de risco para diabetes, sendo mais prevalente em idades avançadas e no sexo feminino. A diabetes gestacional ocorre mais em mulheres que apresentam hipotireoidismo subclínico ${ }^{5}$.

O hipotireoidismo subclínico é uma entidade em que há elevação do nível de TSH (hormônio estimulante da tireoide), mas os níveis de tiroxina sérica livre (T4L) permanecem dentro dos padrões normais. Essa condição pode refletir uma resposta fisiológica do envelhecimento, ou predizer uma disfunção tireoidiana ${ }^{6}$. O hipotireoidismo franco, é chamado de primário, quando há uma disfunção na glândula tireoide, e de secundário, se for de origem hipofisária ou hipotalâmica ${ }^{4,6}$.

A glândula tireoide é responsável pela produção dos hormônios triiodotironina (T3) e tiroxina (T4), que são liberados na circulação sistêmica ligados à proteínas plasmáticas circulantes (transtiretina e globulina ligadora de tiroxina), onde atingem os tecidos periféricos. Nesses tecidos, o T4 é transformado em T3, por ação de enzimas chamadas deiodinases, se ligando aos receptores nucleares nas células com muito mais afinidade do que o T4. Os hormônios tireoidianos têm efeitos sistêmicos no organismo, atuando inclusive no metabolismo, onde aumenta o catabolismo de carboidratos e lipídios e estimula a síntese de proteínas, aumentando o metabolismo basal ${ }^{4,6}$.

Os hormônios tireoidianos estimulam a transcrição de diversos genes e atuam em praticamente todos os tecidos do corpo humano. Dentre as diversas funções desses dois hormônios, é interessante citar algumas em que a diminuição deles está relacionada ao desenvolvimento da DMt2: o T3 e o T4 aumentam as enzimas metabólicas celulares, o que provoca um aumento no metabolismo dos carboidratos, estimulando a absorção de glicose no intestino, a glicólise e gliconeogênese hepática, a captação de glicose e a produção de insulina. Os hormônios tireoidianos também dificultam a deposição de lipídeos no tecido adiposo, aumentando sua concentração plasmática e sua oxidação. Ao mesmo tempo, reduz as concentrações de colesterol e triglicerídeos no plasma ${ }^{5-6}$.

O hipotireoidismo causa a redução das funções descritas acima, havendo uma diminuição da captação de glicose e da produção de insulina, um aumento da deposição de lipídeos no tecido adiposo - e consequente aumento de peso - e uma diminuição de sua oxidação, além de um aumento dos níveis de triglicerídeos plasmáticos. Isso cria um estado de resistência à insulina e de disfunção na produção de insulina, aumentando o risco de desenvolvimento de DMt2 $2^{3,5-8}$.

As duas principais causas de hipotireoidismo são a deficiência de iodo na alimentação e o desenvolvimento de anticorpos contra a glândula tireoide (hipotireoidismo autoimune). O hipotireoidismo autoimune pode ocorrer juntamente com uma insuficiência adrenal autoimune e DMt1, caracterizando a Síndrome Poliendócrina Autoimune Tipo $2^{4}$.

Sendo o hipotireoidismo um fator de risco para diabetes, este estudo teve o objetivo de avaliar a prevalência de hipotireoidismo em pacientes com diabetes mellitus tipo 2 em um Ambulatório de Endocrinologia.

\section{CASUÍSTICA E MÉTODOS}

A pesquisa realizada foi do tipo transversal, analítica e quantitativa, realizada entre julho de 2016 e abril de 2017 , em um ambulatório de endocrinologia de um consultório médico particular.

A amostra foi composta conforme os seguintes critérios de inclusão: pacientes os quais foram atendidos no período de 01 de janeiro de 2015 até 01 de dezembro de 2016 que apresentavam diagnóstico médico de diabetes mellitus tipo 2, prontuários com dados suficientes para categorização (peso, idade, sexo e exames laboratoriais de glicemia, colesterol total e frações, triglicerídeos, TRH, TSH e T3 e T4 livres) e consentimento informado de cada paciente selecionado.

Foram excluídos desta pesquisa os pacientes cujos prontuários médicos estavam com preenchimento incompleto, pacientes que não apresentaram diagnóstico 
médico de diabetes mellitus tipo 2 e os pacientes que não consentiram em participar deste estudo.

O protocolo deste estudo foi realizado segundo as diretrizes éticas da Declaração de Helsinki da Associação Médica Mundial e foi aprovado pelo Comitê de Ética em Pesquisa.

Os instrumentos de coleta foram: ficha clínica com dados sobre perfil e identificação do paciente, inventário de saúde (história médica pessoal e familiar pregressa), aspectos clínicos do motivo da consulta, avaliação física, avaliação laboratorial, diagnóstico médico e plano de tratamento.
Para evitar vieses, foram utilizados prontuários de pacientes que foram atendidos pelo mesmo profissional, com padronização de equipamentos e instrumentos de avaliação física.

O diagnóstico médico de diabetes mellitus tipo 2 foi feito segundo critérios estabelecidos pelas Diretrizes 20142015 da Sociedade Brasileira de Diabetes. O diagnóstico de hipotireoidismo e hipotireoidismo subclínico foram realizados segundo critérios estabelecidos pelas Diretrizes 2005 da Sociedade Brasileira de Endocrinologia. Os critérios para diagnóstico de diabetes mellitus tipo 2 e de hipotireoidismo são apresentados na Tabela 1 .

Tabela 1 Critérios diagnósticos para diabetes mellitus tipo 2 e hipotireoidismo

\begin{tabular}{|c|c|c|c|}
\hline Condições & \multicolumn{3}{|c|}{ Critérios } \\
\hline Diabetes Mellitus tipo 2 & $\begin{array}{l}\text { Sintomas de poliúria, } \\
\text { polidipsia e perda ponderal } \\
\text { acrescidos de glicemia casual } \\
\geq 200 \mathrm{mg} / \mathrm{dl}^{1}\end{array}$ & $\begin{array}{l}\text { Glicemia de jejum } \geq 126 \mathrm{mg} / \mathrm{dl} \\
(7 \mathrm{mmol} / \mathrm{L})^{1}\end{array}$ & $\begin{array}{l}\text { Glicemia de } 2 \text { h pós-sobrecarga } \\
\text { de } 75 \text { g de glicose } \geq 200 \mathrm{mg} / \mathrm{dl}^{1}\end{array}$ \\
\hline Hipotireoidismo & \multicolumn{3}{|c|}{ TSH normal ou elevado $(>5,5 \mathrm{mU} / \mathrm{L})+\mathrm{T}_{4}$ livre $<0,7 \mathrm{ng} / \mathrm{dl}$} \\
\hline
\end{tabular}

1 O diagnóstico de Diabetes Mellitus tipo 2 pode ser feito utilizando-se qualquer um dos três critérios acima.

Foram selecionados 184 prontuários médicos de pacientes atendidos no período entre 01 de janeiro de 2015 e 01 de dezembro de 2016, sendo que foram excluídos 31 pacientes devido à falta de dados necessários para categorização. Foram revisados os prontuários médicos dos 153 pacientes restantes, sendo que apenas 51 atenderam aos critérios de inclusão e foram incluídos nesta pesquisa.

\section{RESULTADOS}

Levando-se em consideração apenas os 51 pacientes diabéticos incluídos neste estudo, 26 eram homens e 25 eram mulheres, sendo que a idade variou entre 15 e 80 anos $(M=56,3)$. Quinze pacientes $(29,4 \%)$ tiveram o diagnóstico de hipotireoidismo (franco ou subclínico). A Tabela 2 mostra os resultados obtidos após análise dos 51 prontuários médicos selecionados.

Tabela 2 - Resultados obtidos após análise dos prontuários médicos

\begin{tabular}{lcc}
\hline Descrição & Valores (mín - máx (média)) \\
\hline TSH (mUI/L) & $0,015-20 \quad(\mathrm{M}=3,28)$ \\
$\mathrm{T}_{4}$ livre (ng/dL) & $0,44-4,66 \quad(\mathrm{M}=1,19)$ \\
Glicemia jejum (mg/dL) & $66-392 \quad(\mathrm{M}=119,95)$ \\
Colesterol total (mg/dL) & $105-322 \quad(\mathrm{M}=183,22)$ \\
Triglicerídeos (mg/dL) & $36-970 \quad(\mathrm{M}=156,74)$ \\
Peso $(\mathrm{kg})$ & $51-148 \quad(\mathrm{M}=74,71)$ \\
\hline
\end{tabular}

A Tabela 3 exibe o número de pacientes diabéticos diagnosticados com hipotireoidismo.

Tabela 3 Pacientes diabéticos com hipotireoidismo

\begin{tabular}{lc}
\hline Diagnóstico & Quantidade de pacientes \\
\hline Hipotireoidismo subclínico & 09 \\
Hipotireoidismo franco & 06 \\
\hline
\end{tabular}

\section{DISCUSSÃO}

A associação entre diabetes mellitus tipo 2 e hipotireoidismo é pouco estudada, mas existem algumas pesquisas que demonstram uma alta prevalência dessa doença tireoidiana em pacientes diabéticos. Pimenta et al. ${ }^{9}$ encontraram uma prevalência de hipotireoidismo de $28,8 \%$ em um estudo com 132 diabéticos, sendo que $80,9 \%$ deles sofriam de diabetes mellitus tipo 2 . Gozzano et al. ${ }^{10}$ estabeleceram uma prevalência de hipotireoidismo de 19,5\% em uma amostra com 118 diabéticos tipo 2.

Chubb et al. ${ }^{11}$ em um estudo envolvendo 420 mulheres com DMt2, encontraram uma prevalência de hipotireoidismo subclínico de 8,6\% . Perros et al. ${ }^{12}$ descobriram uma prevalência de hipotireoidismo de $0,9 \%$ e hipotireoidismo subclínico de 4,8\% em um amplo estudo envolvendo 1310 diabéticos, sendo $69 \%$ deles com DMt2.

Neste estudo, foi determinada uma prevalência de hipotireoidismo (franco ou subclínico) de 29,4\% em 51 
pacientes diabéticos tipo 2, ao passo que a prevalência de hipotireoidismo dentre os 153 pacientes previamente selecionados também foi de $29,4 \%$. Dessa forma, acreditase que a prevalência de hipotireoidismo em pacientes diabéticos tipo 2 não supera a prevalência na população doente que busca atendimento médico endocrinológico.

$\mathrm{Na}$ população geral, por sua vez, Pontes et al. ${ }^{13}$ encontraram uma prevalência de hipotireoidismo de $15,9 \%$ em um grupo com 88 voluntários sem queixas de uma comunidade nordestina brasileira. Em um estudo realizado na Índia com 5360 adultos, Unnikrishnan et al. ${ }^{14}$ encontraram uma prevalência de hipotireoidismo de $10,95 \%$.

Em comunidades suplementadas com iodo, vários estudos pelo mundo determinaram uma prevalência de hipotireoidismo muito baixa na população geral. Vanderpump et al. ${ }^{15}$ em um estudo feito na Inglaterra com 2279 adultos encontraram uma prevalência de $0,33 \%$, sendo que todos os indivíduos afetados eram mulheres. Canaris et al. ${ }^{16}$ estabeleceram uma prevalência de $0,4 \%$ de hipotireoidismo numa população de 25862 adultos na cidade de Colorado (EUA). Em um estudo realizado na Itália com 1411 pessoas maiores de 15 anos em uma comunidade com deficiência de iodo, Aghini-Lombardi et al. ${ }^{17}$ determinaram uma prevalência de hipotireoidismo de $0,2 \%$.

Knudsen et al. ${ }^{18}$ em uma pesquisa realizada na Dinamarca com 2656 pessoas com idades entre 41 e 71 anos em uma comunidade com deficiência de iodo limítrofe, encontraram uma prevalência de hipotireoidismo não diagnosticado de $0,4 \%$. Hollowell et al. ${ }^{19}$ em um estudo realizado por todo o território norte-americano com 16533 pessoas maiores de 12 anos de idade, estabeleceram uma prevalência de hipotireoidismo de $0,2 \%$.

Apesar de que neste estudo não foi demonstrada uma prevalência maior de hipotireoidismo em pacientes com diabetes tipo 2 sobre os demais pacientes que buscam atendimento endocrinológico, o alto valor encontrado sugere que o rastreamento para essa doença pode trazer benefícios para o paciente diabético, na medida em que é possível diagnosticar e tratar a doença precocemente.

\section{CONCLUSÃO}

Os dados confirmam uma elevada prevalência de hipotireoidismo em pacientes com diabetes mellitus tipo 2. Nesse sentido, levando-se em consideração os resultados obtidos neste estudo, sugere-se o rastreamento para hipotireoidismo em todos os pacientes com diabetes tipo 2 .

AGRADECIMENTOS: Agradecemos ao Centro de Especialidades Médicas e Estética - CEM, localizada em Rio Verde, Goiás, pela parceria durante a coleta de dados para a realização da pesquisa.

\section{REFERÊNCIAS}

1. World Health Organization. Diabetes. Geneva: WHO; 2015 [cited 2018 Jan 13\}. Available from: http://www.who.int/ diabetes/en/.

2. Bettencourt JMF. Diabetes mellitus tipo 2 e vitaminas antioxidantes (vitamina E, vitamina C e B-caroteno [Dissertação]. Porto: Universidade do Porto, Faculdade de Ciências da Nutrição e Alimentação; 2010 [citado 11 nov. 2015]. Disponível em: http://hdl.handle.net/10216/54615.

3. Lyssenko V, Jonsson A, Almgren P, Pulizzi N, Isomaa B, Tuomi T, Berglund G, Altshuler D, Nilsson P, Groop L. Clinical risk factors, DNA variants, and the development of type 2 diabetes. N Engl J Med. 2008;359(21):2220-32. doi: 10.1056/NEJMoa0801869.

4. Robbins SL, et al. Robbins \& Cotran: Bases patológicas das doenças. 8a ed. Rio de Janeiro: Elsevier; 2010.

5. Gronich N, Deftereos SN, Lavi I, Persidis AS, Abernethy DR, Rennert G. et al. Hypothyroidism is a risk factor for new-onset diabetes: a cohort study. Diabetes Care. 2015;38(9):1657-64. doi: $10.2337 / \mathrm{dc} 14-2515$.

6. Guyton AC, Hall JE. Tratado de fisiologia médica. 12a ed. Rio de Janeiro: Elsevier; 2011.

7. Silverthorn DU. Fisiologia humana: uma abordagem integrada. 5a ed. Rio de Janeiro: Artmed; 2010.

8. Rauen G, Wachholz PA, Graf H, Pinto MJ. Abordagem do hipotireoidismo subclínico no idoso. Rev Soc Bras Clin Med. 2011;9(4):294-9. Disponível em: http://files.bvs.br/ upload/S/1679-1010/2011/v9n4/a2189.pdf.

9. Pimenta WP, Mazeto GMFS, Callegaro CF, Shibata SA, Marins LV, Yamashita S, et al. Associação de tireopatias em uma população de pacientes com diabetes. Arq Bras Endocrinol Metabol. 2005;49(2):234-40. doi: 10.1590/S000427302005000200009.

10. Gozzano M, Gozzano J, Sugiyama L. Tireopatias em pacientes com diabetes tipo 2. Rev Faculdade Cien Med Sorocaba. 2014;16(Supl). Disponível em: http://revistas.pucsp.br/index. $\mathrm{php} / \mathrm{RFCMS} /$ article/view/21026.

11. Chubb SAP, Davis WA, Inman Z, Davis TM. Prevalence and progression of subclinical hypothyroidism in women with type 2 diabetes: the Fremantle Diabetes Study. Clin Endocrinol. 2005;62(4):480-6. doi: 10.1111/j.1365-2265.2005.02246.x.

12. Perros P, McCrimmon RJ, Shaw G, Frier BM. Frequency of thyroid dysfunction in diabetic patients: value of annual screening. Diabetic Med. 1995;12(7):622-7. doi: 10.1111/ j.1464-5491.1995.tb00553.x.

13. Pontes AAN, Pontes AAN, Adan LF, Costa ADM, Benício AVL, Silva CRA, Morais RM. Prevalência de doenças da tireoide em uma comunidade do nordeste brasileiro. Arq Bras Endocrinol. Metab. 2002;46(5):544-9. doi: 10.1590/S000427302002000500008.

14. Unnikrishnan AG, Kalra S, Sahay RK, Bantwal G, John M, Tewari N. Prevalence of hypothyroidism in adults: 
an epidemiological study in eight cities of India. Indian J Endocrinol Metab. 2013;17(4):647-52. doi: 10.4103/22308210.113755 .

15. Vanderpump MPJ, Tunbridge WM, French JM, Appleton D, Bates D, Clark F, Grimley EJ, Hasan DM, Rodgers H, Tunbridge $\mathrm{F}$, et al. The incidence of thyroid disorders in the community: a twenty-year follow-up of the Whickham survey. Clin Endocrinol (Oxf). 1995;43(1):55-68. doi: 10.1111/j.1365-2265.1995.tb01894.x.

16. Canaris GJ, Manowitz NR, Mayor G, Ridgway EC. The Colorado thyroid disease prevalence study. Arch Intern Med. 2000;160(4):526-34. doi: 10.1001/archinte.160.4.526.

17. Aghini-Lombardi F, Antonangeli L, Martino E, Vitti P, Maccherini D, Leoli F, Rago T, Grasso L, Valeriano R, Balestrieri A, Pinchera A. The spectrum of thyroid disorders in an iodine-deficient community: the Pescopagano survey. J Clin Endocrinol Metab. 1999;84(2):561-6. doi: 10.1210/ jcem.84.2.5508.

18. Knudsen N, Jorgensen T, Rasmussen S, Christiansen E, Perrild $\mathrm{H}$. The prevalence of thyroid dysfunction in a population with borderline iodine deficiency. Clin Endocrinol. 1999;51(3):361-7. doi: 10.1046/j.1365-2265.1999.00816.x.

19. Hollowell JG, Staehling NW, Flanders WD, Hannon WH, Gunter EW, Spencer CA, Braverman LE. Serum TSH, T4, and thyroid antibodies in the United States population (1988 to 1994): National Health and Nutrition Examination Survey (NHANES III). J Clin Endocrinol Metab. 2002;87(2):489-99. doi: $10.1210 /$ jcem.87.2.8182.

Submetido em: 20.02.18

Aceito em: 28.05.18 